\title{
Solution of Some Types for Composition Fractional Order Differential Equations Corresponding to Optimal Control Problems
}

\author{
Sameer Qasim Hasan (D) and Moataz Abbas Holel (D) \\ Department of Mathematics, University of Mustansiriyah, Baghdad, Iraq \\ Correspondence should be addressed to Sameer Qasim Hasan; dr.sameer_kasim@yahoo.com
}

Received 19 February 2018; Revised 9 May 2018; Accepted 28 June 2018; Published 1 August 2018

Academic Editor: Darong Huang

Copyright (C) 2018 Sameer Qasim Hasan and Moataz Abbas Holel. This is an open access article distributed under the Creative Commons Attribution License, which permits unrestricted use, distribution, and reproduction in any medium, provided the original work is properly cited.

\begin{abstract}
The approximate solution for solving a class of composition fractional order optimal control problems (FOCPs) is suggested and studied in detail. However, the properties of Caputo and Riemann-Liouville derivatives are also given with complete details on Chebyshev approximation function to approximate the solution of fractional differential equation with different approach. Also, the relation between Caputo and Riemann-Liouville of fractional derivative took a big role for simplifying the fractional differential equation that represents the constraints of optimal control problems. The approximate solutions are defined on interval $[0,1]$ and are compared with the exact solution of order one which is an important condition to support the working method. Finally, illustrative examples are included to confirm the efficiency and accuracy of the proposed method.
\end{abstract}

\section{Introduction}

The idea of fractional derivative dates back to a conversation between two mathematicians: Leibniz and L'Hopital. In 1695, they exchanged about the meaning of a derivative of order $1 / 2$. Their correspondence has been well documented and is stated as the foundation of fractional calculus [1].

A fractional optimal control problem (FOCPs) is an optimal control problem focused on the performance index or the fractional differential equations governing the dynamics of the system or both contain at least one fractional order derivative term.

The formulation and solution of state and variables FOCPs were first established by Agrawal, where the applied fractional variational calculus (FVC) presented a general formulation and solution scheme for FOCPs in the RiemannLiouville (RL) sense; it was based on variational virtual work coupled with the Lagrange multiplier technique. Since the Caputo fractional derivatives (CFDs) seems more natural and allows incorporating the usual initial conditions, it becomes a popular choice for researchers $[2,3]$.
The Chebyshev polynomials are used to solve composition FOCPs and Chebyshev polynomials $T_{n}(t)$ of degree $n$ are important in approximation theory, since the roots of the Chebyshev polynomials of the first kind, which are also called Chebyshev nodes, are used as nodes in polynomial interpolation.

Agrawal and Baleanu [4] obtained necessary conditions for FOCPs with Riemann-Liouville derivative and then were able to solve the problem numerically.

Elbarbary introduced Chebyshev finite difference approximation for the boundary value problems of integer derivatives [5]. In [6], Khader and Hendy studied an efficient numerical scheme for solving fractional optimal control problems. In [7], Akbarian and Keyanpour studied a new approach to the numerical solution of fractional order optimal control Problems.

This paper presents a new approach for computing the approximate optimal control function for optimal control problems with some types of fractional order differential equation such as multi-fractional order differential equation, and composite fractional order differential equations are 
investigated with first kind Chebyshev approximate polynomial which works very efficiently with fractional orders and optimal control problems. All the discussion and details in addition to the transformations of the steps are given to compute all the useful of the new work. The obtained solutions of the method show that the technique of the approach is very convenient and efficient, and many calculations give high accuracy and may lead to near to exact solution by changing up the order of fractional positive integer numbers.

The multi-fractional differential equations corresponding to optimal control problems and basic theorems have been given with algorithm for multi-composite fractional order optimal control problems. Also, we give illustrative examples for the solution of the approximate systems.

The value of $x(t)$ is observed in detail to explain the activity of solution approximation with mixed boundary conditions. It is important to notice that the calculations are written by using the mathematical software MATHCAD by version (14.0.3.332).

This paper consists of the following sections: in Section 2, some basic definitions and properties of fractional order calculus are introduced (R-L and Caputo fractional derivatives). In Section 3, the shifted Chebyshev polynomials and numerical approximations of CFD and RLFD using Chebyshev polynomials are introduced. In Section 4, we derive the necessary optimality conditions of composition order fractional optimal control problems. In Section 5, we give numerical examples to solve composition FOCPs and show the accuracy of the presented method. Finally, conclusions are presented in Section 6.

\section{Preliminaries}

In this section, we give some definitions and properties of fractional derivatives that will be needed later on.

\subsection{Fractional Order Calculus}

Definition 1 (see [8]). The left (LRLFD) and right (RRLFD) Riemann-Liouville fractional derivatives of a function $f(t)$ are defined as

$$
{ }_{a} D_{t}^{\alpha} f(t)=\frac{1}{\Gamma(n-\alpha)} \frac{d^{n}}{d t^{n}} \int_{a}^{t}(t-\tau)^{n-\alpha-1} f(\tau) d \tau,
$$

$$
t>a
$$

$$
\begin{aligned}
& { }_{t} D_{b}^{\alpha} f(t) \\
& \quad=\frac{1}{\Gamma(n-\alpha)}(-1)^{n} \frac{d^{n}}{d t^{n}} \int_{t}^{b}(\tau-t)^{n-\alpha-1} f(\tau) d \tau,
\end{aligned}
$$

$$
t<b \text {. }
$$

where the order of the derivative $\alpha$ satisfies $n-1<\alpha \leq n$, and $\Gamma(n)$ is the gamma function.
Definition 2 (see [8]). The left (LCFD) and right (RCFD) Caputo fractional derivatives of a function $f(t)$ are defined as

$$
\begin{aligned}
&{ }_{a}^{C} D_{t}^{\alpha} f(t)=\frac{1}{\Gamma(n-\alpha)} \int_{a}^{t}(t-\tau)^{n-\alpha-1} f^{(n)}(\tau) d \tau .(\alpha>0, t>0) \\
&{ }_{t}^{C} D_{b}^{\alpha} f(t)=\frac{(-1)^{n}}{\Gamma(n-\alpha)} \int_{t}^{b}(\tau-t)^{n-\alpha-1} f^{(n)}(\tau) d \tau,
\end{aligned}
$$

where $n-1<\alpha \leq n, n \in N$.

2.2. Some Properties of the Fractional Calculus Are Presented in Detail Which Will Be Needed Later on. (i) The fractional operators are linear [9]:

$$
P[\lambda f(t)+\mu g(t)]=\lambda P f(t)+\mu P g(t),
$$

where $P$ is one of ${ }_{a} D_{t}^{\alpha},{ }_{t} D_{b}^{\alpha},{ }_{a}^{C} D_{t}^{\alpha},{ }_{t}^{C} D_{b}^{\alpha},{ }_{a} D_{t}^{-\alpha}$ or ${ }_{t} D_{b}^{-\alpha}$ and $\lambda$, $\mu$ are real numbers.

(ii) Let $0<\alpha<1, n-1<\alpha+\beta<n, n-1<\beta<$ $n, n$ is a positive integer, $f \in C^{n}[a, t]$, and if $x^{(i)}(0)=0, i=$ $0,1, \ldots . . n-1).[9]$, then

$$
{ }_{a}^{C} D_{t}^{\alpha}\left({ }_{a}^{C} D_{t}^{\beta} x(t)\right)={ }_{a}^{C} D_{t}^{\beta}\left({ }_{a}^{C} D_{t}^{\alpha} x(t)\right)={ }_{a}^{C} D_{t}^{\alpha+\beta} x(t) .
$$

(iii) If $0<\alpha<1$, we obtain the following [2]:

$$
\begin{aligned}
\int_{a}^{b} g(t) \cdot{ }_{a}^{C} D_{t}^{\alpha} f(t) d t= & \int_{a}^{b} f(t) \cdot{ }_{t} D_{b}^{\alpha} g(t) d t \\
& +\left[{ }_{t} I_{b}^{1-\alpha} g(t) \cdot f(t)\right]_{a}^{b} \\
\int_{a}^{b} g(t) \cdot{ }_{t}^{C} D_{b}^{\alpha} f(t) d t= & \int_{a}^{b} f(t) \cdot{ }_{a} D_{t}^{\alpha} g(t) d t \\
& +\left[{ }_{a} I_{t}^{1-\alpha} g(t) \cdot f(t)\right]_{a}^{b} .
\end{aligned}
$$

Moreover, if $f$ is a function such that $f(a)=f(b)=0$, we have simpler formulas:

$$
\begin{aligned}
& \int_{a}^{b} g(t) \cdot{ }_{a}^{C} D_{t}^{\alpha} f(t) d t=\int_{a}^{b} f(t) \cdot{ }_{t} D_{b}^{\alpha} g(t) d t, \\
& \int_{a}^{b} g(t) \cdot{ }_{t}^{C} D_{b}^{\alpha} f(t) d t=\int_{a}^{b} f(t) \cdot{ }_{a} D_{t}^{\alpha} g(t) d t .
\end{aligned}
$$

(iv) The relation between the RLFD and the CFD is as follows [2].

For $\alpha>0$ and $n=[\alpha]+1$, the Riemann-Liouville and Caputo fractional derivatives are related by the following formulas:

$$
\begin{aligned}
& { }_{a} D_{t}^{\alpha} f(t)={ }_{a}^{C} D_{t}^{\alpha} f(t)+\sum_{k=0}^{n-1} \frac{f^{(k)}(a)}{\Gamma(k+1-\alpha)}(t-a)^{k-\alpha}, \\
& { }_{t} D_{b}^{\alpha} f(t)={ }_{t}^{C} D_{b}^{\alpha} f(t)+\sum_{k=0}^{n-1} \frac{f^{(k)}(b)}{\Gamma(k+1-\alpha)}(b-t)^{k-\alpha} .
\end{aligned}
$$


(v) The constant function and power function of Caputo's derivative are as follows:

(1) ${ }_{a}^{C} D_{t}^{\alpha} C=0$, where $C$ is a constant.

In particular, ${ }_{a}^{C} D_{t}^{\alpha} 1=0$, and, also from right integral, ${ }_{t}^{C} D_{b}^{\alpha} 1=0$.

(2) ${ }_{a}^{C} D_{t}^{\alpha}(t-a)^{\beta}$

$$
= \begin{cases}0, & \text { for } \beta \in N_{0} \text { and } \beta<\lceil\alpha\rceil, \\ \frac{\Gamma(\beta+1)}{\Gamma(\beta-\alpha+1)}(t-a)^{\beta-\alpha}, & \text { for } \beta \in N_{0} \text { and } \beta \geq\lceil\alpha\rceil,\end{cases}
$$

where $\lceil\alpha\rceil$ denotes the smallest integer greater than or equal to $\alpha$ and $N_{0}=\{0,1,2, \ldots\}$. Recall that for $\alpha \in N$, [10].

\section{The Shifted Chebyshev Polynomials}

The well-known Chebyshev polynomials are defined on the interval $[-1,1]$ and can be determined by the following recurrence formula [11]:

$$
\begin{aligned}
& T_{n}(t)=2 t T_{n-1}(t)-T_{n-2}(t), \\
& T_{0}(t)=1, \\
& T_{1}(t)=t,
\end{aligned}
$$

$$
n=2,3 \ldots
$$

The Chebyshev polynomials can be expanded in power series as follows:

$$
T_{n}(t)=\frac{n}{2} \sum_{m=0}^{\lfloor n / 2\rfloor}(-1)^{m} \frac{(n-m-1) !}{(m) !(n-2 m) !}(2 t)^{n-2 m},
$$

$n=0,1,2, \ldots, m=0,1,2, \ldots,\lfloor n / 2\rfloor$ and $\lfloor n / 2\rfloor$ denotes the biggest integral less than or equal to $n / 2$.

The Chebyshev polynomials $T_{n}(t)(n=0,1,2, \ldots)$ are orthogonal under integration over $[-1,1]$ with the weighting function $w(t)=1 / \sqrt{1-t^{2}}$, with orthogonal condition:

$$
\int_{-1}^{1} \frac{T_{i}(t) T_{j}(t)}{\sqrt{1-t^{2}}} d t= \begin{cases}0 & \text { if } i \neq j, \\ \frac{\pi}{2} & \text { if } i=j \neq 0 \\ \pi & \text { if } i=j=0 .\end{cases}
$$

where $i, j=0,1,2 \ldots$.

In order to use these polynomials on the interval $[0, L]$ we define the so-called shifted pseudo-spectral Chebyshev polynomials by introducing the change of variable $z=2 t / L-$ 1. [12]:

The shifted Chebyshev polynomials are defined as follows

$$
T_{n}^{p}(t)=T_{n}\left(\frac{2 t}{L}-1\right)
$$

$$
\text { where } T_{0}^{p}(t)=1, T_{1}^{p}(t)=\frac{2 t}{L}-1 \text {. }
$$

The analytic form of the shifted pseudo-spectral Chebyshev polynomial $T_{n}^{p}(t)$ of degree $n$ is given by the following [10]:

$$
T_{n}^{p}(t)=n \sum_{k=0}^{n}(-1)^{n-k} \frac{2^{2 k}(n+k-1) !}{(2 k) !(n-k) ! L^{k}} t^{k},
$$

$$
n=1,2 \ldots
$$

where $T_{n}^{p}(0)=(-1)^{n}$, and $T_{n}^{p}(L)=1$.

The orthogonality relation is as follows:

$$
\int_{0}^{L} T_{n}^{p}(t) T_{m}^{p}(t) w^{p}(t) d t=\delta m
$$

with the weight function $w^{p}(t)=1 / \sqrt{L t-t^{2}}, \delta m=$ $\left(b_{k} / 2\right) \pi, b_{0}=2, b_{k}=1$ for $k \geq 1$.

The function $x(t) \in L^{2}([0, L])$ can be expressed in terms of shifted Chebyshev polynomials as follows:

$$
x(t)=\sum_{j=0}^{\infty} c_{n} T_{n}^{p}(t),
$$

where the coefficients $c_{n}$ are given by

$$
c_{n}=\frac{1}{\delta n} \int_{0}^{L} x(t) T_{n}^{p}(t) w^{p}(t) d t, \quad n=0,1, \ldots
$$

3.1. The Chebyshev-Gauss-Lobatto Points. We choose the Chebyshev-Gauss-Lobatto points associated with the interval $[0, L]$, as follows:

$$
t_{r}=\frac{L}{2}-\frac{L}{2} \cos \left(\frac{\pi r}{N}\right), \quad r=0,1, \ldots N .
$$

These grids can be written as $L<x_{N}<x_{N-1} \ldots<x_{1}<x_{0}=$ 0 .

Clenshaw and Curtis [13] introduced an approximation of the function $x(t)$, as follows:

$$
\begin{aligned}
x_{N}(t) & =\sum_{n=0}^{N} a_{n}^{\prime \prime} T_{n}^{p}(t) \\
a_{n} & =\frac{2}{N} \sum_{r=0}^{N \prime} x\left(t_{r}\right) T_{n}^{p}\left(t_{r}\right) .
\end{aligned}
$$

where $\left({ }^{\prime \prime}\right)$ on the summation means that the first and last terms are to be taken with a factor $(1 / 2)$. 
Theorem 3 (see [2]). The fractional derivative of order $\alpha$ in the Caputo sense for the function $x(t)$ at the point $t_{s}$ is given by

$$
{ }_{0}^{C} D_{t}^{\alpha} x_{N}\left(t_{s}\right)=\sum_{r=0}^{N} d_{s, r}^{(\alpha)} x\left(t_{r}\right), \quad \alpha>0,
$$

Such that

$$
\begin{aligned}
d_{s, r}^{(\alpha)} & =\frac{4 \theta_{r}}{N} \sum_{n=\lceil\alpha\rceil}^{N} \sum_{j=0}^{N} \sum_{k=\lceil\alpha\rceil}^{n} \frac{n \theta_{n}}{b_{j}} \\
\cdot & \frac{(-1)^{n-k}(n+k-1) ! \Gamma(k-\alpha+1 / 2) T_{n}^{p}\left(t_{r}\right) T_{j}^{p}\left(t_{s}\right)}{L^{\alpha} \Gamma(k+1 / 2)(n-k) ! \Gamma(k-\alpha-j+1) \Gamma(k-\alpha+j+1)},
\end{aligned}
$$

where

$$
\begin{aligned}
s, r & =0,1,2, \ldots, N \text { and } \\
\theta_{0} & =\theta_{N}=\frac{1}{2}, \\
\theta_{i} & =1 \quad \forall i=1,2, \ldots, N-1 .
\end{aligned}
$$

Proof. The fractional derivative of the approximate formula for the function $x(t)$ in (22) is given by

$$
\begin{aligned}
& { }_{0}^{C} D_{t}^{\alpha} x_{N}(t)=\sum_{n=0}^{N}{ }^{\prime \prime} a_{n}{ }_{0}^{C} D_{t}^{\alpha} T_{n}^{p}(t) \\
& { }_{0}^{C} D_{t}^{\alpha} x_{N}(t) \\
& =\sum_{n=0}^{N}{ }^{\prime \prime}{ }_{n}{ }_{0}^{C} D_{t}^{\alpha}\left(n \sum_{k=0}^{n}(-1)^{n-k} \frac{2^{2 k}(n+k-1) !}{(2 k) !(n-k) ! L^{k}} t^{k}\right)
\end{aligned}
$$

$$
{ }_{0}^{C} D_{t}^{\alpha} T_{n}^{p}(t)=\sum_{j=0}^{N} \sum_{k=\lceil\alpha\rceil}^{n} \frac{(-1)^{n-k} 2 n(n+k-1) ! \Gamma(k-\alpha+1 / 2) T_{j}^{p}(t)}{b_{j} L^{\alpha} \Gamma(k+1 / 2)(n-k) ! \Gamma(k-\alpha-j+1) \Gamma(k-\alpha+j+1)} .
$$

From (29) and (32), we have

$$
{ }_{0}^{C} D_{t}^{\alpha} x_{N}(t)=\frac{4}{N} \sum_{n=\lceil\alpha\rceil}^{N} \sum_{r=0}^{\prime \prime} \sum_{j=0}^{N} \sum_{k=\lceil\alpha\rceil}^{n} \frac{(-1)^{n-k} n(n+k-1) ! \Gamma(k-\alpha+1 / 2) x\left(t_{r}\right) T_{n}^{p}\left(t_{r}\right) T_{j}^{p}(t)}{b_{j} L^{\alpha} \Gamma(k+1 / 2)(n-k) ! \Gamma(k-\alpha-j+1) \Gamma(k-\alpha+j+1)} .
$$

From (33), the fractional derivative of order $\alpha$ for the function $x(t)$ at the point $t_{s}$ leads to the desired result.

Theorem 4 (see [2]). Let ${ }_{0}^{C} D_{t}^{\alpha} x_{N}(t)$ be the approximation of the fractional derivative ${ }_{0}^{C} D_{t}^{\alpha}$ of the function $x(t)$ as given by (24). Then it holds
Using (12) and (13), in (18) we have

$$
{ }_{0}^{C} D_{t}^{\alpha} T_{n}^{p}(t)=0, \quad n=0,1, \ldots,\lceil\alpha\rceil-1,
$$

then

$$
{ }_{0}^{C} D_{t}^{\alpha} x_{N}(t)=\sum_{n=\lceil\alpha\rceil}^{N}{ }^{\prime \prime} a_{n}^{C} D_{t}^{\alpha} T_{n}^{p}(t)
$$

Therefore, for $n=\lceil\alpha\rceil,\lceil\alpha+1\rceil, \ldots, N$ and by (12) and (13), in for formula (18), we get

$$
\begin{aligned}
& { }_{0}^{C} D_{t}^{\alpha} T_{n}^{p}(t)=n \sum_{k=0}^{n}(-1)^{n-k} \frac{2^{2 k}(n+k-1) !}{(2 k) !(n-k) ! L^{k}}{ }_{0}^{C} D_{t}^{\alpha} t^{k} \\
& \quad=n \sum_{k=\lceil\alpha\rceil}^{n}(-1)^{n-k} \frac{2^{2 k}(n+k-1) ! k !}{(2 k) !(n-k) ! L^{k} \Gamma(k-\alpha+1)} t^{k-\alpha} .
\end{aligned}
$$

Now, $t^{k-\alpha}$ can be expressed approximately in terms of shifted pseudo-spectral Chebyshev polynomial series, so we have

$$
t^{k-\alpha} \cong \sum_{j=0}^{N} a_{k j} T_{j}^{p}(t)
$$

where $a_{k j}$ is obtained from (21) with $x(t)=t^{k-\alpha}$. If only the first $(N+1)$-terms from pseudo-spectral shifted Chebyshev polynomials in (23) are considered, the approximate formula for the fractional derivative of the shifted pseudo-spectral Chebyshev polynomials is as follows: 
where

$$
\begin{aligned}
\Omega_{n} & =\sum_{k=[\alpha]}^{n} \frac{(-1)^{n-k} 2 n(n+k-1) ! \Gamma(k-\alpha+1 / 2)}{b_{j} L^{\alpha} \Gamma(k+1 / 2)(n-k) ! \Gamma(k-\alpha-j+1) \Gamma(k-\alpha+j+1)}, \\
F\left(x ; y_{1}, y_{2}, \ldots y_{n}\right) & =\left|\begin{array}{cccc}
\langle x, x\rangle & \left\langle x, y_{1}\right\rangle & \cdots & \left\langle x, y_{n}\right\rangle \\
\left\langle y_{1}, x\right\rangle & \left\langle y_{1}, y_{1}\right\rangle & \cdots & \left\langle y_{1}, y_{n}\right\rangle \\
\vdots & \vdots & \ddots & \vdots \\
\left\langle y_{n} ; x\right\rangle & \left\langle y_{n} ; y_{1}\right\rangle & \cdots & \left\langle y_{n} ; y_{n}\right\rangle
\end{array}\right|
\end{aligned}
$$

3.2. Approximation of the Right Riemann-Liouville Fractional Derivative. Using the right Caputo fractional derivative (RCFD) of a function $f(t)$ is defined in (4) when $0<\alpha<1$ and $f(t) \in A C[a, b]$,

$$
{ }_{s}^{c} D_{b}^{\alpha} f(s)=\frac{-1}{\Gamma(1-\alpha)} \int_{s}^{b}(t-s)^{-\alpha} f^{\prime}(t) d t,
$$

From relation between the RLFD and the CFD (11), when $0<$ $\alpha<1$, (that is $n=1$ ), we have

$$
{ }_{s} D_{b}^{\alpha} f(s)=\frac{f(b)}{\Gamma(1-\alpha)}(b-s)^{-\alpha}+{ }_{s}^{c} D_{b}^{\alpha} f(s),
$$

Use (37) in (38) to obtain

$$
\begin{aligned}
{ }_{s} D_{b}^{\alpha} f(s)= & \frac{f(b)}{\Gamma(1-\alpha)}(b-s)^{-\alpha} \\
& +\frac{1}{\Gamma(1-\alpha)} \int_{s}^{b}(t-s)^{-\alpha} f^{\prime}(t) d t
\end{aligned}
$$

Let $f$ be a sufficiently smooth function in $[0, b]$ and let $J(s ; f)$ be defined as follows:

$$
J(s ; f)=\int_{s}^{b}(t-s)^{-\alpha} f^{\prime}(t) d t, \quad 0<s<b .
$$

Substituting (40) in (39), we deduce

$$
{ }_{s} D_{b}^{\alpha} f(s)=\frac{f(b)}{\Gamma(1-\alpha)}(b-s)^{-\alpha}+\frac{J(s ; f)}{\Gamma(1-\alpha)} .
$$

Now, we approximate $f(t), 0 \leq t \leq b$, by a sum of shifted Chebyshev polynomials $T_{k}(2 t / b-1)$ according to

$$
\begin{aligned}
& f(t) \approx p_{N}(t)=\sum_{k=0}^{N}{ }^{\prime \prime} a_{k} T_{k}\left(\frac{2 t}{b}-1\right), \\
& a_{k}=\frac{2}{N} \sum_{j=0}^{N \prime} f\left(t_{j}\right) T_{k}\left(\frac{2 t_{j}}{b}-1\right),
\end{aligned}
$$

where

$$
\begin{gathered}
t_{j}=\frac{b}{2}-\frac{b}{2} \cos \left(\frac{\pi j}{N}\right), \\
\quad j=0, \ldots, N, \text { and obtain } \\
J(s ; f) \approx J\left(s ; p_{N}\right)=\int_{s}^{b} p_{N}{ }^{\prime}(t)(t-s)^{-\alpha} d t .
\end{gathered}
$$

Lemma 5 (see [7]). Let $p_{N}$ be the polynomial of degree $N$ as given by (42); then there exists a polynomial $\mathrm{F}_{\mathrm{N}-1}$ of degree $\mathrm{N}-$ 1 such that

$$
\begin{aligned}
& \int_{s}^{x}\left[p_{N}^{\prime}(t)-p_{N}^{\prime}(s)\right](t-s)^{-\alpha} d t \\
& \quad=\left[F_{N-1}(x)-F_{N-1}(s)\right](x-s)^{1-\alpha} .
\end{aligned}
$$

Proof. Let $p_{N}{ }^{\prime}(t)-p_{N}{ }^{\prime}(s)$ be expanded in a Taylor series at $t=s$

$$
p_{N}^{\prime}(t)-p_{N}^{\prime}(s)=\sum_{k=1}^{N-1} A_{k}(s)(t-s)^{k}
$$

Then,

$$
\begin{aligned}
\int_{s}^{x} & {\left[p_{N}{ }^{\prime}(t)-p_{N}{ }^{\prime}(s)\right](t-s)^{-\alpha} d t } \\
& =\sum_{k=1}^{N-1} A_{k}(s) \int_{s}^{x}(t-s)^{k-\alpha} d t \\
& =\left[(t-s)^{1-\alpha} \sum_{k=1}^{N-1} \frac{A_{k}(s)(t-s)^{k}}{k-\alpha+1}\right]_{s}^{x} .
\end{aligned}
$$

The assertion follows, if we choose $F_{N-1}(x)=$ $\sum_{k=0}^{N-1}\left(A_{k}(s)(x-s)^{k} /(k-\alpha+1)\right)$, with an arbitrary constant $A_{0}(s)$. From (44), we have

$$
\begin{aligned}
& J\left(s ; p_{N}\right)=\int_{s}^{b} p_{N}{ }^{\prime}(t)(t-s)^{-\alpha} d t \\
& \quad=\left[\frac{p_{N}^{\prime}(s)}{1-\alpha}+\left[F_{N-1}(b)-F_{N-1}(s)\right]\right](b-s)^{1-\alpha}
\end{aligned}
$$


Moreover, ${ }_{s} D_{b}^{\alpha} f(s)$ can be approximated by means of

$$
{ }_{s} D_{b}^{\alpha} f(s) \approx \frac{f(b)}{\Gamma(1-\alpha)}(b-s)^{-\alpha}+\frac{J\left(s ; p_{N}\right)}{\Gamma(1-\alpha)} .
$$

We express $F_{N-1}(t)$ in (47) by a sum of Chebyshev polynomials and provide the recurrence relation satisfied by the Chebyshev coefficients. Differentiating both sides of (44) with respect to $x$ yields

$$
\begin{aligned}
& {\left[p_{N}{ }^{\prime}(x)-p_{N}^{\prime}(s)\right](x-s)^{-\alpha}} \\
& =F_{N-1}^{\prime}(x)(x-s)^{1-\alpha} \\
& \quad+\left[F_{N-1}(x)-F_{N-1}(s)\right](1-\alpha)(x-s)^{-\alpha} . \\
& p_{N}^{\prime}(x)-p_{N}^{\prime}(s) \\
& =F_{N-1}^{\prime}(x)(x-s) \\
& \quad+\left[F_{N-1}(x)-F_{N-1}(s)\right](1-\alpha) .
\end{aligned}
$$

To evaluate $F_{N-1}(s)$ in (47), we expand $F_{N-1}{ }^{\prime}(x)$ in terms of the shifted Chebyshev polynomials:

$$
F_{N-1}^{\prime}(x)=\sum_{k=0}^{N-2} b_{k} T_{k}\left(\frac{2 x}{b}-1\right), \quad 0 \leq x \leq b,
$$

where $\left(^{\prime}\right)$ on the summation means that the first term is to be taken with a factor $(1 / 2)$.

$$
\begin{aligned}
& F_{N-1}(x)-F_{N-1}(s) \\
& \quad=\frac{b}{4} \sum_{k=1}^{N-1} \frac{b_{k-1}-b_{k+1}}{k}\left(T_{k}\left(\frac{2 x}{b}-1\right)-T_{k}\left(\frac{2 s}{b}-1\right)\right),
\end{aligned}
$$

where $b_{N-1}=b_{N}=0$. On the other hand, we have

$$
\begin{aligned}
& (x-s) F_{N-1}^{\prime}(x) \\
& =\frac{b}{2} F_{N-1}^{\prime}(x)\left[\left(\frac{2 x}{b}-1\right)-\left(\frac{2 s}{b}-1\right)\right] .
\end{aligned}
$$

By using the relation $T_{k+1}(v)+T_{k-1}(v)=2 v T_{k}(v)$ and from (51), it follows that

$$
\begin{aligned}
& (x-s) F_{N-1}^{\prime}(x)=\frac{b}{4} \\
& \cdot \sum_{k=1}^{N-1^{\prime}}\left(b_{k+1}-2\left(\frac{2 s}{b}-1\right) b_{k}+b_{k-1}\right) T_{k}\left(\frac{2 x}{b}-1\right),
\end{aligned}
$$

Such that $b_{-1}=b_{1}$.

Let

$$
p_{N}^{\prime}(x)=\sum_{k=0}^{N-1} c_{k} T_{k}\left(\frac{2 x}{b}-1\right)
$$

Inserting $F_{N-1}(x)-F_{N-1}(s)$ and $(x-s) F_{N-1}{ }^{\prime}(x)$ as given (52) and (54) into (50) and taking (55) into account, we get

$$
\begin{aligned}
& \left(1-\frac{1-\alpha}{k}\right) b_{k+1}-2\left(\frac{2 s}{b}-1\right) b_{k}+\left(1+\frac{1-\alpha}{k}\right) b_{k-1} \\
& =\frac{4}{b} c_{k}, \quad 1 \leq k .
\end{aligned}
$$

The Chebyshev coefficients $c_{k}$ of $p_{N}{ }^{\prime}(x)$ as given by (55) can be evaluated by integrating and comparing it with (42):

$$
c_{k-1}=c_{k+1}+\frac{4 k}{b} a_{k}, \quad k=N, N-1, \ldots, 1,
$$

with starting values $c_{N}=c_{N+1}=0$, where $a_{k}$ are the Chebyshev coefficients of $p_{N}(x)$.

\section{The Necessary Optimality Conditions of Composition Order Fractional Optimal Control Problems

$$
M_{a}^{C} D_{t}^{\alpha}(t)+N{ }_{a}^{C} D_{t}^{\beta}\left({ }_{a}^{C} D_{t}^{\mu} x(t)\right)=f(t, x(t), u(t))
$$

In this problem, the constraint has multiorder and composition for the three fractional derivatives $\alpha, \beta$, and $\mu$, where $\alpha, \beta$ and $\mu>0$. The multi-order fractional optimal control problems refers to the minimization of an objective functional subject to dynamical constraints on the state and the control which have three fractional derivatives order models.

The necessary optimality conditions of this type are introduced as follows.

Let $\alpha, \beta$ and $\mu$ be real numbers and $\alpha, \beta$ and $\mu \in(0,1)$, and let $f_{0}$ and $f$ be two differentiable functions with domain $\left[a, \infty\left[\times \mathbb{R}^{2}\right.\right.$. We consider a general form of multicomposition fractional optimal control problem:

$$
\mathrm{J}(x, u, T)=\int_{a}^{T} f_{0}(t, x(t), u(t)) d t
$$

which subject to the multi derivative-composite fractional dynamic control system,

$$
M_{a}^{C} D_{t}^{\alpha}(t)+N{ }_{a}^{C} D_{t}^{\beta}\left({ }_{a}^{C} D_{t}^{\mu} x(t)\right)=f(t, x(t), u(t)),
$$

and the boundary conditions,

$$
\begin{gathered}
x(a)=x_{a}, \\
x(T)=x_{T} .
\end{gathered}
$$

where $M, N \neq 0, T, x_{a}$ and $x_{T}$ are fixed real numbers.

Theorem 6. If $(x, u, T)$ is a minimizer of (58), (59), and (60), then there exists a function $\lambda(t)$ for which $(x, u, T)$ satisfies the multi-composite fractional optimality conditions: 
(i) The Hamiltonian system is defined as

$$
\begin{gathered}
M_{t} D_{T}^{\alpha} \lambda(t)+N_{t} D_{T}^{\beta}\left({ }_{t} D_{T}^{\mu} \lambda(t)\right) \\
=\frac{\partial H}{\partial x}(t, x(t), u(t), \lambda(t)) \\
M_{a}^{C} D_{t}^{\alpha} x(t)+N{ }_{a}^{C} D_{t}^{\beta}\left({ }_{a}^{C} D_{t}^{\mu} x(t)\right) \\
=\frac{\partial H}{\partial \lambda}(t, x(t), u(t), \lambda(t)) .
\end{gathered}
$$

(ii) The stationary condition is

$$
\frac{\partial H}{\partial u}(t, x(t), u(t), \lambda(t))=0 \quad \text { For all } t \in[a, T]
$$

Proof. We consider the following multi-composite fractional optimal control problem:

$$
\begin{aligned}
& \operatorname{minimize} J(x, u, T)=\int_{a}^{T} f_{0}(t, x(t), u(t)) d t, \\
& M_{a}^{C} D_{t}^{\alpha}(t)+N{ }_{a}^{C} D_{t}^{\beta}\left({ }_{a}^{C} D_{t}^{\mu} x(t)\right)=f(t, x(t), u(t)),
\end{aligned}
$$

Thus,

$$
\begin{aligned}
J^{*} & (x, u, T, \lambda)=\int_{a}^{T}\left\{f_{0}(t, x(t), u(t))+\lambda(t)\right. \\
& \cdot[f(t, x(t), u(t)) \\
& \left.\left.-\left(M_{a}^{C} D_{t}^{\alpha}(t) x(t)+N{ }_{a}^{C} D_{t}^{\beta}\left({ }_{a}^{C} D_{t}^{\mu} x(t)\right)\right)\right]\right\} d t
\end{aligned}
$$

Suppose that

$$
\begin{aligned}
F= & f_{0}(t, x(t), u(t))+\lambda(t) f(t, x(t), u(t)) \\
& -M \lambda(t){ }_{a}^{C} D_{t}^{\alpha}(t) x(t)-N \lambda(t){ }_{a}^{C} D_{t}^{\beta}\left({ }_{a}^{C} D_{t}^{\mu} x(t)\right)
\end{aligned}
$$

Using (8) in (65), we get

$$
\begin{aligned}
F= & f_{0}(t, x(t), u(t))+\lambda(t) f(t, x(t), u(t)) \\
& -M x(t){ }_{t} D_{T}^{\alpha} \lambda(t)-N x(t){ }_{t} D_{T}^{\beta}\left({ }_{t} D_{T}^{\mu} \lambda(t)\right),
\end{aligned}
$$

Evaluating the Euler equation for $x(t)$ of (66),

$$
\begin{aligned}
& \frac{\partial F}{\partial x(t)}-\frac{d}{d t}\left(\frac{\partial F}{\partial x^{\cdot}(t)}\right)=0, \\
& \frac{\partial f_{0}}{\partial x(t)}+\lambda(t) \frac{\partial f}{\partial x(t)}-M_{t} D_{T}^{\alpha} \lambda(t) \\
& -N_{t} D_{T}^{\beta}\left({ }_{t} D_{T}^{\mu} \lambda(t)\right)-\frac{d}{d t}\left(\frac{\partial F}{\partial x^{\cdot}(t)}\right)=0
\end{aligned}
$$

From (67), we get $(d / d t)\left(\partial F / \partial x^{\prime}(t)\right)=0$, as follows:

$$
\begin{gathered}
\frac{\partial f_{0}}{\partial x(t)}+\lambda(t) \frac{\partial f}{\partial x(t)}-M_{t} D_{T}^{\alpha} \lambda(t) \\
-N_{t} D_{T}^{\beta}\left({ }_{t} D_{T}^{\mu} \lambda(t)\right)=0
\end{gathered}
$$

Now, evaluating Euler's equation for $\lambda(t)$ of (65):

$$
\begin{gathered}
\frac{\partial F}{\partial \lambda(t)}-\frac{d}{d t}\left(\frac{\partial F}{\partial \lambda \cdot(t)}\right)=0 \\
\frac{\partial f_{0}}{\partial \lambda(t)}+\lambda(t) \frac{\partial f}{\partial \lambda(t)}-M_{a}^{C} D_{t}^{\alpha}(t) x(t) \\
-N{ }_{a}^{C} D_{t}^{\beta}\left({ }_{a}^{C} D_{t}^{\mu} x(t)\right)=0
\end{gathered}
$$

Finally, the Euler's equation for $u(t)$ of $(65)$ is

$$
\frac{\partial F}{\partial u(t)}-\frac{d}{d t}\left(\frac{\partial F}{\partial u^{\prime}(t)}\right)=0 .
$$

From (65) it is clear that $(d / d t)\left(\partial F / \partial u^{\prime}(t)\right)=0$, then we get

$$
\frac{\partial F}{\partial u(t)}=\frac{\partial f_{0}}{\partial u(t)}+\lambda(t) \frac{\partial f}{\partial u(t)}=0
$$

From (69), (71), and (73) we have that

$$
\begin{aligned}
& M_{t} D_{T}^{\alpha} \lambda(t)+N_{t} D_{T}^{\beta}\left({ }_{t} D_{T}^{\mu} \lambda(t)\right) \\
& \quad=\frac{\partial H}{\partial x}(t, x(t), u(t), \lambda(t)) \\
& M_{a}^{C} D_{t}^{\alpha}(t) x(t)+N{ }_{a}^{C} D_{t}^{\beta}\left({ }_{a}^{C} D_{t}^{\mu} x(t)\right) \\
& \quad=\frac{\partial H}{\partial \lambda}(t, x(t), u(t), \lambda(t)) \\
& 0=\frac{\partial H}{\partial u}(t, x(t), u(t), \lambda(t)) \quad \text { For all } t \in[a, T] .
\end{aligned}
$$

\section{Illustrative Example}

In this section, we consider the following linear-quadratic of multi-composite fractional optimal control problem:

$$
\min J(x, u)=\int_{0}^{1}(u(t)-x(t))^{2} d t
$$

which is subject to the multi-composite fractional dynamical system,

$$
\begin{aligned}
& { }_{0}^{C} D_{t}^{\alpha} x(t)+{ }_{0}^{C} D_{t}^{\beta}\left({ }_{0}^{C} D_{t}^{\mu} x(t)\right) \\
& =u(t)-x(t)+\frac{6 t^{\alpha+1}}{\Gamma(\alpha+2)}+\frac{6 t^{\beta+\mu+1}}{\Gamma(\beta+\mu+2)},
\end{aligned}
$$


and the boundary conditions,

$$
\begin{aligned}
& x(0)=0, \\
& x(1)=\frac{6}{\Gamma(\alpha+\beta+\mu+2)} .
\end{aligned}
$$

The exact solution for $\alpha=\beta=\mu=1$ is given by the following:

$$
\bar{x}(t)=\bar{u}(t)=\frac{6 t^{\alpha+\beta+\mu+1}}{\Gamma(\alpha+\beta+\mu+2)} .
$$

Now, we develop algorithm for solution (75), (76), and (77). It is based on the necessary optimality conditions from Theorem 6 and implements the following steps.

Step 1. Compute the Hamiltonian function:

$$
\begin{aligned}
H & =(u(t)-x(t))^{2}+\lambda(t) \\
& \cdot\left(u(t)-x(t)+\frac{6 t^{\alpha+1}}{\Gamma(\alpha+2)}+\frac{6 t^{\beta+\mu+1}}{\Gamma(\beta+\mu+2)}\right) .
\end{aligned}
$$

Step 2. Derive the necessary optimality conditions from Theorem 6:

$$
\begin{aligned}
& { }_{t} D_{1}^{\alpha} \lambda(t)+{ }_{t} D_{1}^{\beta}\left({ }_{t} D_{1}^{\mu} \lambda(t)\right)=\frac{\partial H}{\partial x} \\
& =-(2(u(t)-x(t))+\lambda(t)), \\
& { }_{0}^{C} D_{t}^{\alpha} x(t)+{ }_{0}^{C} D_{t}^{\beta}\left({ }_{0}^{C} D_{t}^{\mu} x(t)\right)=\frac{\partial H}{\partial \lambda} \\
& =u(t)-x(t)+\frac{6 t^{\alpha+1}}{\Gamma(\alpha+2)}+\frac{6 t^{\beta+\mu+1}}{\Gamma(\beta+\mu+2)}, \\
& 2(u(t)-x(t))+\lambda(t)=0 .
\end{aligned}
$$

Step 3. Find the coupled system.

(i) From substitution (82) in (80), we get

$$
{ }_{t} D_{1}^{\alpha} \lambda(t)+{ }_{t} D_{1}^{\beta}\left({ }_{t} D_{1}^{\mu} \lambda(t)\right)=0 .
$$

(ii) Find control variable $u(t)$ from (82) and substitution in (80) to obtain

$$
\begin{aligned}
& { }_{0}^{C} D_{t}^{\alpha} x(t)+{ }_{0}^{C} D_{t}^{\beta}\left({ }_{0}^{C} D_{t}^{\mu} x(t)\right) \\
& =-\frac{1}{2} \lambda(t)+\frac{6 t^{\alpha+1}}{\Gamma(\alpha+2)}+\frac{6 t^{\beta+\mu+1}}{\Gamma(\beta+\mu+2)},
\end{aligned}
$$

Using property (6) for (83) and (84), we get the coupled system:

$$
\begin{aligned}
& { }_{t} D_{1}^{\alpha} \lambda(t)+{ }_{t} D_{1}^{\beta+\mu} \lambda(t)=0, \\
& { }_{0}^{C} D_{t}^{\alpha} x(t)+{ }_{0}^{C} D_{t}^{\beta+\mu} x(t) \\
& \quad=-\frac{1}{2} \lambda(t)+\frac{6 t^{\alpha+1}}{\Gamma(\alpha+2)}+\frac{6 t^{\beta+\mu+1}}{\Gamma(\beta+\mu+2)} .
\end{aligned}
$$

Step $4 a$. Solve (85a) by Chebyshev expansion method, and use (48) to approximate $\lambda(t)$.

$$
\begin{aligned}
{ }_{t} D_{1}^{\alpha} \lambda(t) \approx & \frac{\lambda(1)}{\Gamma(1-\alpha)}\left(1-t_{s}\right)^{-\alpha}+\frac{J\left(t_{s} ; p_{N}\right)}{\Gamma(1-\alpha)} \\
{ }_{t} D_{1}^{\beta+\mu} \lambda(t) \approx & \frac{\lambda(1)}{\Gamma(1-(\beta+\mu))}\left(1-t_{s}\right)^{-(\beta+\mu)} \\
& +\frac{J\left(t_{s} ; p_{N}\right)}{\Gamma(1-(\beta+\mu))},
\end{aligned}
$$

$s=1,2, \ldots, N-1$, where $J\left(t_{s} ; p_{N 1}\right)$ and $J\left(t_{s} ; p_{N 2}\right)$ is defined in (47).

Sum both (86) and (87) to get

$$
\begin{aligned}
\lambda(1) & {\left[\frac{\left(1-t_{s}\right)^{-\alpha}}{\Gamma(1-\alpha)}+\frac{\left(1-t_{s}\right)^{-(\beta+\mu)}}{\Gamma(1-(\beta+\mu))}\right]+\frac{J\left(t_{s} ; p_{N 1}\right)}{\Gamma(1-\alpha)} } \\
& +\frac{J\left(t_{s} ; p_{N 2}\right)}{\Gamma(1-(\beta+\mu))}=0 .
\end{aligned}
$$

$s=1,2, \ldots, N-1$, where $J\left(t_{s} ; p_{N 1}\right)$ and $J\left(t_{s} ; p_{N 2}\right)$ is defined in (47).

Step $4 b$. Solve ( $85 \mathrm{~b}$ ) by Chebyshev expansion method, and use (24) to approximate $x(t)$.

$$
\begin{aligned}
{ }_{0}^{C} D_{t}^{\alpha} x_{N}\left(t_{s}\right) & =\sum_{r=0}^{N} d_{s, r}^{(\alpha)} x\left(t_{r}\right), \\
{ }_{0}^{C} D_{t}^{\beta+\mu} x_{N}\left(t_{s}\right) & =\sum_{r=0}^{N} d_{s, r}^{(\beta+\mu)} x\left(t_{r}\right), \quad \alpha,(\beta+\mu)>0 .
\end{aligned}
$$

$s, r=0,1,2, \ldots, N$, where $d_{s, r}^{(\alpha)}$ is defined in (25).

Using (89) and (90) in (85a) to get

$$
\begin{aligned}
& \sum_{r=0}^{N} d_{s, r}^{(\alpha)} x\left(t_{r}\right)+\sum_{r=0}^{N} d_{s, r}^{(\beta+\mu)} x\left(t_{r}\right) \\
& =-\frac{1}{2} \lambda\left(t_{s}\right)+\frac{6 t_{s}^{\alpha+1}}{\Gamma(\alpha+2)}+\frac{6 t_{s}^{\beta+\mu+1}}{\Gamma(\beta+\mu+2)} .
\end{aligned}
$$

where $d_{s, r}^{(\alpha)}$ and $d_{s, r}^{(\beta+\mu)}$ are defined in (25).

Step 5. Find the results at the shifted Gauss-Lobatto nodes $t_{s}$, from (22) when $s=1,2, \ldots, N-1$, and $\alpha, \beta$ and $\mu \in(0,1)$, in two cases.

Case 1. Let $N=2, \alpha=0.4, \beta=0.2$ and $\mu=0.1 \longrightarrow \beta+\mu=$ 0.3 .

$s=1,2, \ldots, N-1$, and since $N=2$, then $s=1 . t_{1}=$ $1 / 2-(1 / 2) \cos (\pi / 2)=0.5$. 
TABLE 1: The values of Caputo fractional derivative when $(\alpha=0.4, \beta=0.2$ and $\mu=0.1$ in Case 1$)$ and $(\alpha=0.7, \beta=0.4$ and $\mu=0.3$ in Case 2 needed later on) are shown.

\begin{tabular}{cllcccccc}
\hline$n$ & $j$ & $k$ & $d_{1,1}^{(0.4)}$ & $d_{1,1}^{(0.3)}$ & $d_{1,2}^{(0.4)}$ & $d_{1,2}^{(0.3)}$ & $d_{1,1}^{(0.7)}$ & $d_{1,2}^{(0.7)}$ \\
\hline 1 & 0 & 1 & 0 & 0 & 0.6722 & 0.6274 & 0 & 0.8154 \\
1 & 1 & 1 & 0 & 0 & 0 & 0 & 0 \\
1 & 2 & 1 & 0 & 0 & 0.0387 & 0.0287 & 0 \\
2 & 0 & 1 & 0.6722 & 0.6274 & -0.6722 & -0.6274 & 0.8154 & -0.8154 \\
2 & 1 & 1 & 0 & 0 & 0 & 0 & 0 \\
2 & 2 & 1 & 0.0387 & 0.0287 & -0.0387 & -0.0287 & 0.0572 \\
2 & 0 & 2 & -0.5777 & -0.5210 & 0.5777 & 0.5210 & -0.7720 & 0.0572 \\
2 & 1 & 2 & 0 & 0 & 0 & 0 & 0 \\
2 & 2 & 2 & 0.0592 & 0.0620 & -0.0592 & -0.0620 & 0.0396 \\
& sum & & 0.1924 & 0.1971 & 0.5185 & 0.459 & 0.1402 & 0.0396 \\
& $d_{s, r}^{(\alpha)}=4 \theta_{r} /$ N.sum & & 0.3848 & 0.3942 & 0.5185 & 0.459 & 0.7324 \\
\hline
\end{tabular}

Now, substituting the value of $\alpha, \beta, \mu$ and $t_{1}$, in (88), we get

$$
\begin{gathered}
\lambda(1)[1.8346]+(0.6715) J\left(t_{s} ; p_{N 1}\right) \\
+(0.7704) J\left(t_{s} ; p_{N 2}\right)=0
\end{gathered}
$$

where $s=1,2, \ldots, N-1$, where $J\left(t_{s} ; p_{N 1}\right)$ and $J\left(t_{s} ; p_{N 2}\right)$ are defined in (47).

After calculating (92) at the shifted Gauss-Lobatto nodes $t_{s}=t_{1}=0.5$ and substitution in (88) we get $\lambda\left(t_{1}\right)=0$.

Now, we calculate (91) and, using the boundary conditions (78), we have

$$
\begin{aligned}
d_{1,0}^{(0.4)} & x\left(t_{0}\right)+d_{1,1}^{(0.4)} x\left(t_{1}\right)+d_{1,2}^{(0.4)} x\left(t_{2}\right)+d_{1,0}^{(0.3)} x\left(t_{0}\right) \\
& +d_{1,1}^{(0.3)} x\left(t_{1}\right)+d_{1,2}^{(0.3)} x\left(t_{2}\right) \\
= & -\frac{1}{2} \lambda\left(t_{1}\right)+\frac{6 t_{1}^{1.4}}{\Gamma(2.4)}+\frac{6 t_{s}^{1.3}}{\Gamma(2.3)} .
\end{aligned}
$$

Use (25) to evaluate the computation of $d_{1,1}^{(0.4)}, d_{1,2}^{(0.4)}, d_{1,1}^{(0.3)}$ and $d_{1,2}^{(0.3)}$, which is shown in Table 1 .

when $r=1 \longrightarrow 4 \theta_{1} / N=4(1) / 2=2$ and $r=2 \longrightarrow$ $4 \theta_{2} / N=4(0.5) / 2=1$.

The results of Table 1 and boundary conditions from (78) can be substituted in (93); we get

$$
\begin{aligned}
& d_{1,0}^{(0.4)} x(0)+(0.3848) x\left(t_{1}\right)+(0.5185) x(1) \\
& +d_{1,0}^{(0.3)} x(0)+(0.3942) x\left(t_{1}\right) \\
& \quad+(0.459) x(1)=\frac{6 t_{1}^{1.4}}{\Gamma(2.4)}+\frac{6 t_{1}^{1.3}}{\Gamma(2.3)} \\
& \quad d_{1,0}^{(0.4)}(0)+(0.3848) x\left(t_{1}\right)+(0.5185)\left(\frac{6}{\Gamma(2.7)}\right) \\
& \quad+d_{1,0}^{(0.3)}(0)+(0.3942) x\left(t_{1}\right) \\
& \quad+(0.459)\left(\frac{6}{\Gamma(2.7)}\right)=\frac{6(0.5)^{1.4}}{\Gamma(2.4)}+\frac{6(0.5)^{1.3}}{\Gamma(2.3)} \\
& x\left(t_{1}\right)=0.1566 .
\end{aligned}
$$

Step 6. Compute approximation solution of $x(t)$ and $u(t)$ at $N=2$.

$$
\begin{aligned}
& x_{2}(t)=\frac{2}{N} \sum_{n=0}^{N} \sum_{r=0}^{N \prime} x\left(t_{r}\right) T_{n}^{p}\left(t_{r}\right) T_{n}^{p}(t), \\
& x_{2}(t)=\frac{1}{2} a_{0} T_{0}^{p}(t)+a_{1} T_{1}^{p}(t)+\frac{1}{2} a_{2} T_{2}^{p}(t) .
\end{aligned}
$$

To find $a_{0}, a_{1}$ and $a_{2}$ from (23), we get

$$
\begin{aligned}
& \text { If } n=0 \longrightarrow \\
& a_{0}=\sum_{r=0}^{2} x\left(t_{r}\right) T_{0}^{p}\left(t_{r}\right) \text {, i.e. } T_{0}^{p}(t)=1 \text {. } \\
& =2.0204 \text {. } \\
& \text { If } n=1 \longrightarrow \\
& a_{1}=\sum_{r=0}^{2} x\left(t_{r}\right) T_{1}^{p}\left(t_{r}\right), \quad \text { i.e. } T_{1}^{p}(t)=\frac{2 t}{b}-1 \\
& =1.9421 \text {. }
\end{aligned}
$$

If $n=2 \longrightarrow$

$$
\begin{aligned}
a_{2} & =\sum_{r=0}^{2} x\left(t_{r}\right) T_{2}^{p}\left(t_{r}\right), \\
& =1.8638 .
\end{aligned}
$$

Now, we substitute $a_{0}, a_{1}$, and $a_{2}$, in (98), to get

$$
x_{2}(t)=7.4552 t^{2}-3.571 t \text {. }
$$

To compute the approximation solution of the control $u(t)$ from (76), we get

$$
\begin{aligned}
u(t)= & { }_{0}^{C} D_{t}^{\alpha} x(t)+{ }_{0}^{C} D_{t}^{\beta}\left({ }_{0}^{C} D_{t}^{\mu} x(t)\right) \\
& +x(t)-\frac{6 t^{\alpha+1}}{\Gamma(\alpha+2)} \\
& -\frac{6 t^{\beta+\mu+1}}{\Gamma(\beta+\mu+2)},
\end{aligned}
$$


TABLE 2: The numerical values of the state $x(t)$ and control $u(t)$ with $N=2$ which converged to exact values are shown.

\begin{tabular}{|c|c|c|c|c|c|c|}
\hline \multirow{3}{*}{$t$} & $\alpha=0.4$ & $\alpha=0.7$ & $\alpha=1$ & $\alpha=0.4$ & $\alpha=0.7$ & $\alpha=1$ \\
\hline & $\beta=0.2$ & $\beta=0.4$ & $\beta=1$ & $\beta=0.2$ & $\beta=0.4$ & $\beta=1$ \\
\hline & $\mu=0.1$ & $\mu=0.3$ & $\mu=1$ & $\mu=0.1$ & $\mu=0.3$ & $\mu=1$ \\
\hline 0 & 0 & 0.0001 & 0 & 0 & 0.0001 & 0 \\
\hline 0.1 & -0.283 & 0.1966 & 0.078 & -2.0660 & 0.5152 & 0.78 \\
\hline 0.2 & -0.416 & 0.3942 & 0.0252 & -2.9336 & 0.1920 & 0.252 \\
\hline 0.3 & -0.4 & 0.5928 & 0.502 & -2.9336 & 0.2078 & 0.502 \\
\hline 0.4 & -0.236 & 0.7925 & 0.818 & -3.0727 & 0.6427 & 0.818 \\
\hline 0.5 & 0.078 & 0.9932 & 1.196 & -2.4852 & 1.0983 & 1.196 \\
\hline 0.6 & 0.541 & 1.1950 & 1.63 & -1.5024 & 1.5673 & 1.63 \\
\hline 0.7 & 1.153 & 1.3978 & 2.118 & -0.1432 & 2.0459 & 2.118 \\
\hline 0.8 & 1.915 & 1.6017 & 2.658 & -0.1432 & 2.5314 & 2.658 \\
\hline 0.9 & 2.825 & 1.8067 & 3.247 & -0.1432 & 3.0221 & 3.247 \\
\hline 1 & 3.884 & 2.0127 & 3.884 & 6.0665 & 3.5170 & 3.884 \\
\hline
\end{tabular}

$$
\begin{aligned}
{ }_{0}^{C} D_{t}^{0.4} x_{2}(t) & =10.4290 t^{1.6}-3.9963 t^{0.6} . \\
{ }_{0}^{C} D_{t}^{0.2}\left({ }_{0}^{C} D_{t}^{0.1} x_{2}(t)\right) & =9.6527 t^{1.7}-3.9301 t^{0.7} .
\end{aligned}
$$

Using (102), (103), and (100) in (101), we have:

$$
\begin{aligned}
u(t)= & 10.4290 t^{1.6}-3.9963 t^{0.6}+9.6527 t^{1.7} \\
& -3.9301 t^{0.7}+7.4552 t^{2}-3.571 t \\
& -4.8303 t^{1.4}-5.1427 t^{1.3}
\end{aligned}
$$

Case 2. Let $N=2, \alpha=0.7, \beta=0.4$ and $\mu=0.3 \longrightarrow \beta+\mu=$ 0.7 .

$s=1,2, \ldots, N-1$, and since $N=2$, then $s=1 . t_{1}=$ $1 / 2-(1 / 2) \cos (\pi / 2)=0.5$.

Now, we calculate (91) and using the boundary conditions (78), we have

$$
\begin{aligned}
d_{1,0}^{(0.7)} x & \left(t_{0}\right)+d_{1,1}^{(0.7)} x\left(t_{1}\right)+d_{1,2}^{(0.7)} x\left(t_{2}\right)+d_{1,0}^{(0.7)} x\left(t_{0}\right) \\
& +d_{1,1}^{(0.7)} x\left(t_{1}\right)+d_{1,2}^{(0.7)} x\left(t_{2}\right) \\
= & -\frac{1}{2} \lambda\left(t_{1}\right)+\frac{6 t_{1}^{1.7}}{\Gamma(2.7)}+\frac{6 t_{s}^{1.7}}{\Gamma(2.7)}
\end{aligned}
$$

To find $d_{1,1}^{(0.7)}$ and $d_{1,2}^{(0.7)}$ from Table 1 and we get $\lambda\left(t_{1}\right)=0$, in the same steps of Case 1.

$$
\begin{aligned}
& d_{1,0}^{(0.7)}(0)+(0.2804) x\left(t_{1}\right)+(0.7324)(2.0126) \\
& \quad+d_{1,0}^{(0.7)}(0)+(0.2804) x\left(t_{1}\right) \\
& \quad+(0.7324)(2.0126)=2.3911, \\
& x\left(t_{1}\right)=0.9932 .
\end{aligned}
$$

Now, compute approximation solution of $x(t)$ and $u(t)$ at $N=$ 2, in Case 2:

$$
\begin{aligned}
& x_{2}(t)=\frac{2}{N} \sum_{n=0}^{N} \sum_{r=0}^{N \prime \prime} x\left(t_{r}\right) T_{n}^{p}\left(t_{r}\right) T_{n}^{p}(t), \\
& x_{2}(t)=\frac{1}{2} a_{0} T_{0}^{p}(t)+a_{1} T_{1}^{p}(t)+\frac{1}{2} a_{2} T_{2}^{p}(t) .
\end{aligned}
$$

To find $a_{0}, a_{1}$ and $a_{2}$, from (23), we get

$$
\begin{aligned}
& a_{0}=1.9995, \\
& a_{1}=1.0063 \text { and } \\
& a_{2}=0.0131 .
\end{aligned}
$$

We substitute $a_{0}, a_{1}$, and $a_{2}$, in (108), to get

$$
x_{2}(t)=0.0528 t^{2}+1.9598 t+0.0001 \text {. }
$$

To compute the approximation solution of the control $u(t)$, we get

$$
\begin{aligned}
& u(t)={ }_{0}^{C} D_{t}^{\alpha} x(t)+{ }_{0}^{C} D_{t}^{\beta}\left({ }_{0}^{C} D_{t}^{\mu} x(t)\right)+x(t) \\
&-\frac{6 t^{\alpha+1}}{\Gamma(\alpha+2)}-\frac{6 t^{\beta+\mu+1}}{\Gamma(\beta+\mu+2)}, \\
&{ }_{0}^{C} D_{t}^{0.7} x_{2}(t)= 0.0905 t^{1.3}+2.1837 t^{0.3}
\end{aligned}
$$

Now, using (110) and (112) in (111), we get

$$
\begin{aligned}
u(t)= & 0.0905 t^{1.3}+2.1837 t^{0.3}+0.0528 t^{2}+1.9598 t \\
& +0.0001-3.8843 t^{1.7} .
\end{aligned}
$$

In Table 2, we give the computation of $x(t)$ and $u(t)$ when values $t=0 \ldots 1$ with different values of orders $\alpha, \beta$ and $\mu$, for fractional derivative ( $\alpha=0.4, \beta=0.2$, and $\mu=0.1$.); see Figures $1(\mathrm{~b})$ and 2(b) and ( $\alpha=0.7, \beta=0.4$, and $\mu=0.3$ ); see Figures $1(\mathrm{c})$ and $2(\mathrm{c})$ for $N=2$, which converge to exact values with $(\alpha=\beta=\mu=1)$; see Figures 1(a) and 2(a). 


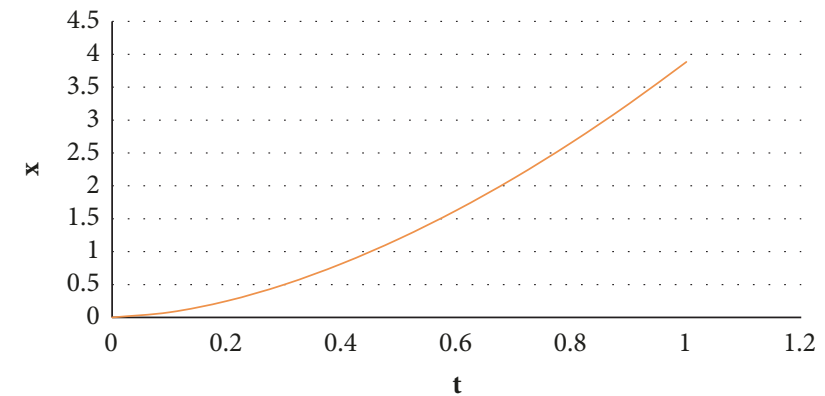

(a) Exact solution of $x(t)$ for $(N=2)$ and $\mu=\alpha=\beta=1$.

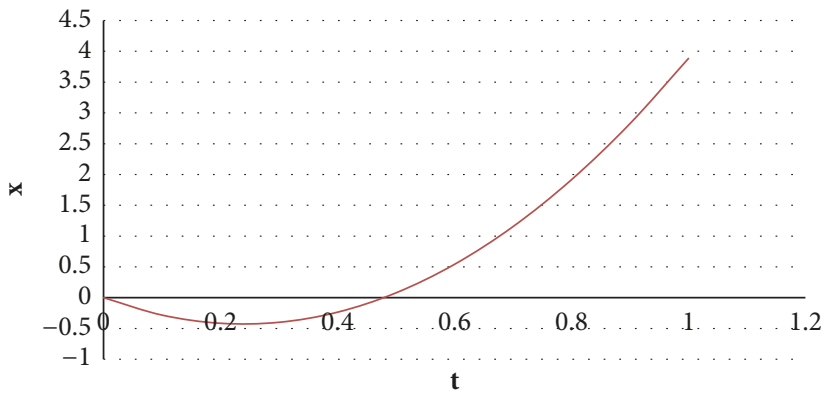

(b) Approximate solutions of $x(t)$ with $(N=2)$ and $\alpha=0.4, \beta=$ 0.2 and $\mu=0.1$

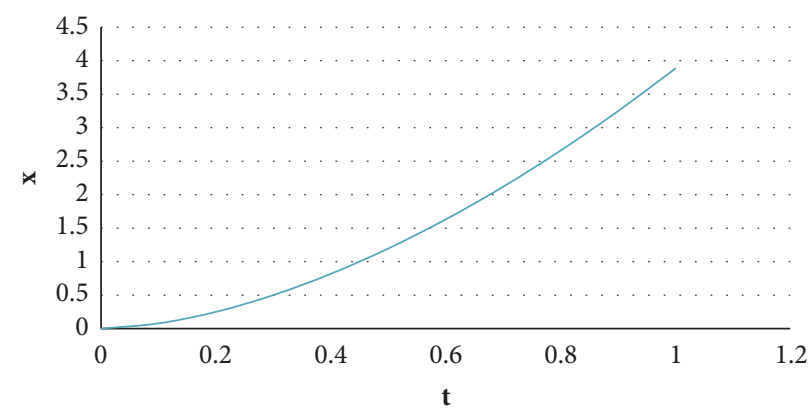

(c) Approximate solutions of $x(t)$ with $(N=2) \alpha=0.7, \beta=$ 0.4 and $\mu=0.3$.

FIGURE 1: Display the exact and approximate values of state $\mathrm{x}(\mathrm{t})$ with different types of $\alpha, \beta, \mu$.

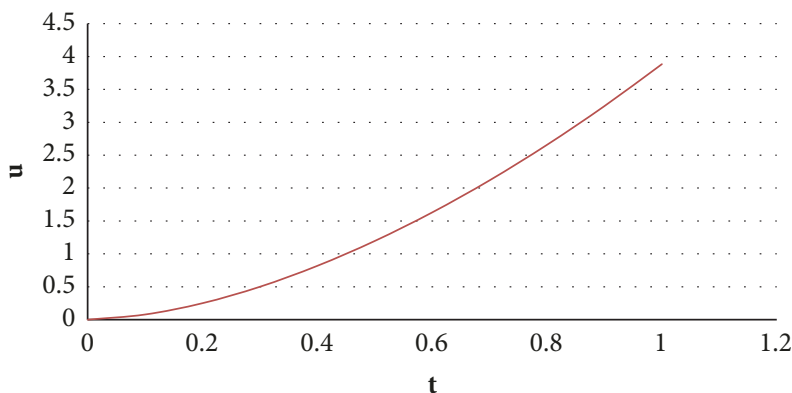

(a) Exact solution of $u(t)$ with $(N=2)$ and $\alpha=\beta=\mu=1$.

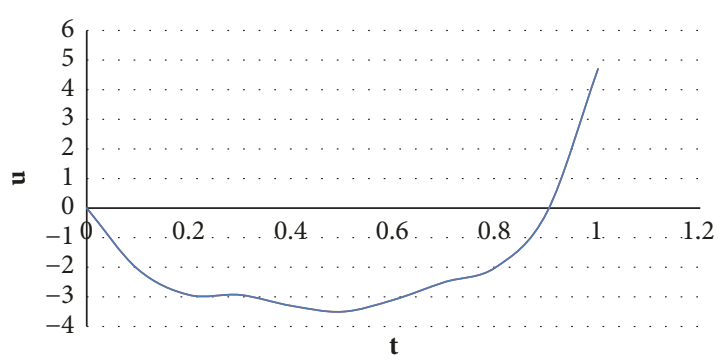

(b) Approximate solutions of $u(t)$ for $(N=2)$ and $\alpha=0.4, \beta=$ 0.2 and $\mu=0.1$

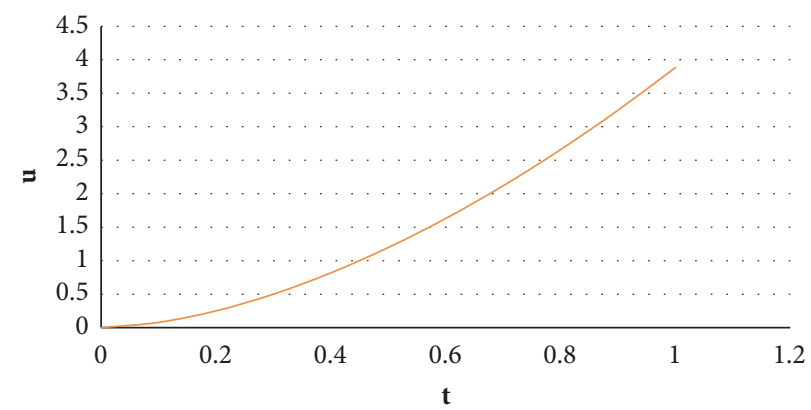

(c) Approximate solutions of $u(t)$ with $(N=2) \alpha=0.7, \beta=$ 0.4 and $\mu=0.3$.

FIGURE 2: Display the exact and approximate values of control input $\mathrm{u}($.$) with different types of \alpha, \beta, \mu$. 


\section{Conclusions}

In this paper, we have presented algorithm for solution a class of multi-composite order fractional optimal control problems, in two cases one when $\alpha=0.4, \beta=0.2$ and $\mu=$ 0.1 , and the other one when $\alpha=0.7, \beta=0.4$ and $\mu=0.3$ at $(N=2)$. In both cases, the solution is approximated by Chebyshev series. Numerical results for illustrative example show that the algorithm converges from the exact solution when $\alpha=\beta=\mu=1$, and we note that the convergent to the exact solution is dependent on increasing of the fractional order of derivative.

\section{Conflicts of Interest}

The authors declare that they have no conflicts of interest.

\section{References}

[1] K. Oldham and J. Spanier, The fractional calculus theory and applications of differentiation and integration to arbitrary order, vol. 111, Elsevier, 1974.

[2] R. Almeida, S. Pooseh, and D. F. Torres, Computational methods in the fractional calculus of variations, World Scientific Publishing Co Inc, 2015.

[3] C. Tricaud and Y. Q. Chen, "An approximate method for numerically solving fractional order optimal control problems of general form," Computers \& Mathematics with Applications, vol. 59, no. 5, pp. 1644-1655, 2010.

[4] O. P. Agrawal and D. Baleanu, "A hamiltonian formulation and a direct numerical scheme for fractional optimal control problems," Journal of Vibration and Control, vol. 13, no. 9-10, pp. 1269-1281, 2007.

[5] E. M. Elbarbary and M. El-Kady, "Chebyshev finite difference approximation for the boundary value problems," Applied Mathematics and Computation, vol. 139, no. 2-3, pp. 513-523, 2003.

[6] M. M. Khader and A. Hendy, "An efficient numerical scheme for solving fractional optimal control problems," International Journal of Nonlinear Science, vol. 14, pp. 287-296.

[7] T. Akbarian and M. Keyanpour, "A new approach to the numerical solution of fractional order optimal control problems," Applications and Applied Mathematics. An International Journal, vol. 8, no. 2, pp. 523-534, 2013.

[8] Y.Zhou, Basic Theory of Fractional Differential Equations, World Scientific, Singapore, 2014.

[9] A. B. Malinowska and D. F. Torres, Introduction to the fractional calculus of variations, World Scientific Publishing Co Inc, 2012.

[10] Q. M. Al-Mdallal, M. I. Syam, and M. N. Anwar, "A collocationshooting method for solving fractional boundary value problems," Communications in Nonlinear Science and Numerical Simulation, vol. 15, no. 12, pp. 3814-3822, 2010.

[11] N. H. Sweilam and M. M. Khader, "A Chebyshev pseudospectral method for solving fractional-order integrodifferential equations," Anziam Journal, vol. 51, no. 4, pp. 464-475, 2010.

[12] B. Obsieger, Numerical Methods III-Approximation of Functions, University-Books, 2013.

[13] C. W. Clenshaw and A. R. Curtis, "A method for numerical integration of an automatic computer," Numerische Mathematik, vol. 2, no. 1, pp. 197-205, 1960. 


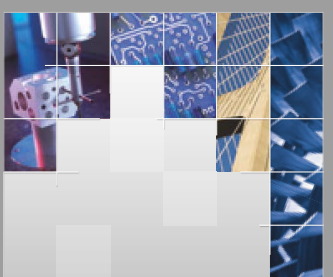

\section{Enfincering}
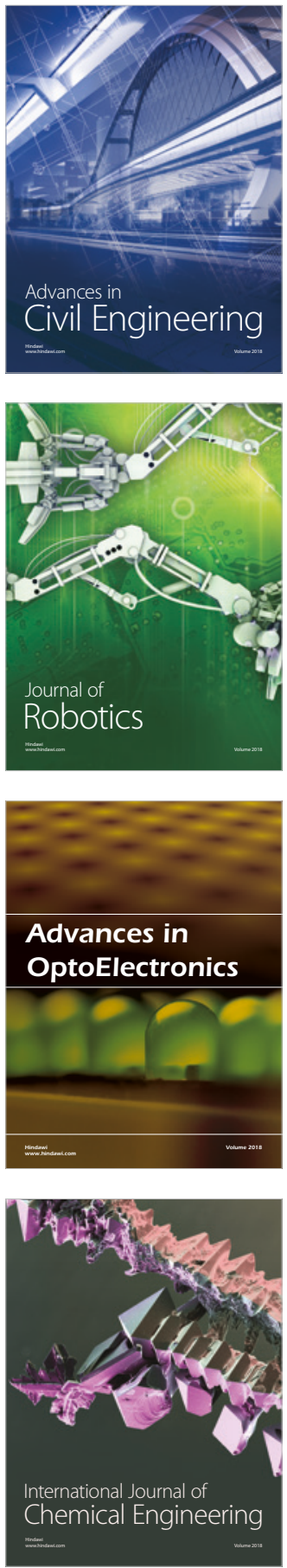

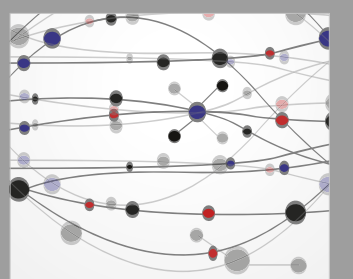

\section{Rotating \\ Machinery}

The Scientific World Journal

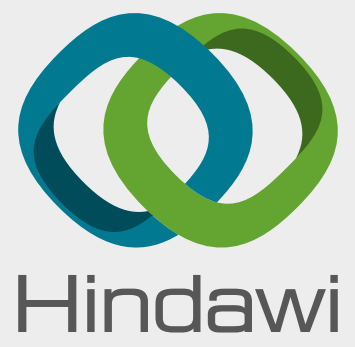

Submit your manuscripts at

www.hindawi.com
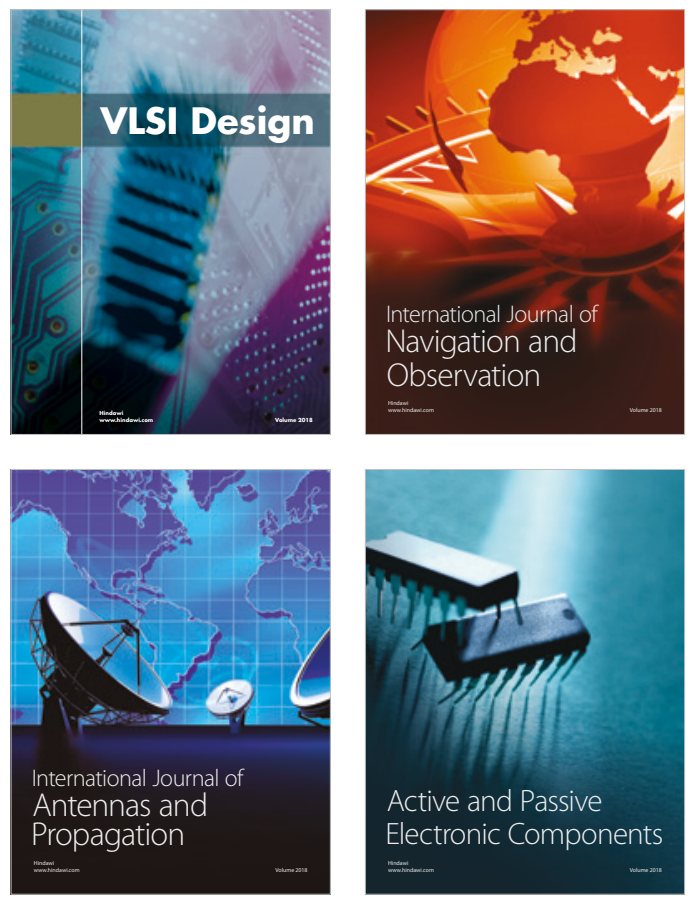
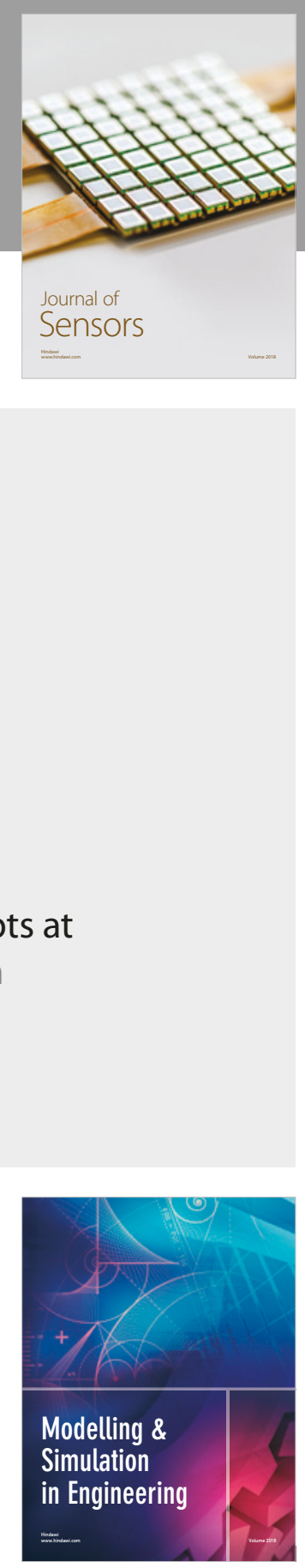

\section{Advances \\ Multimedia}
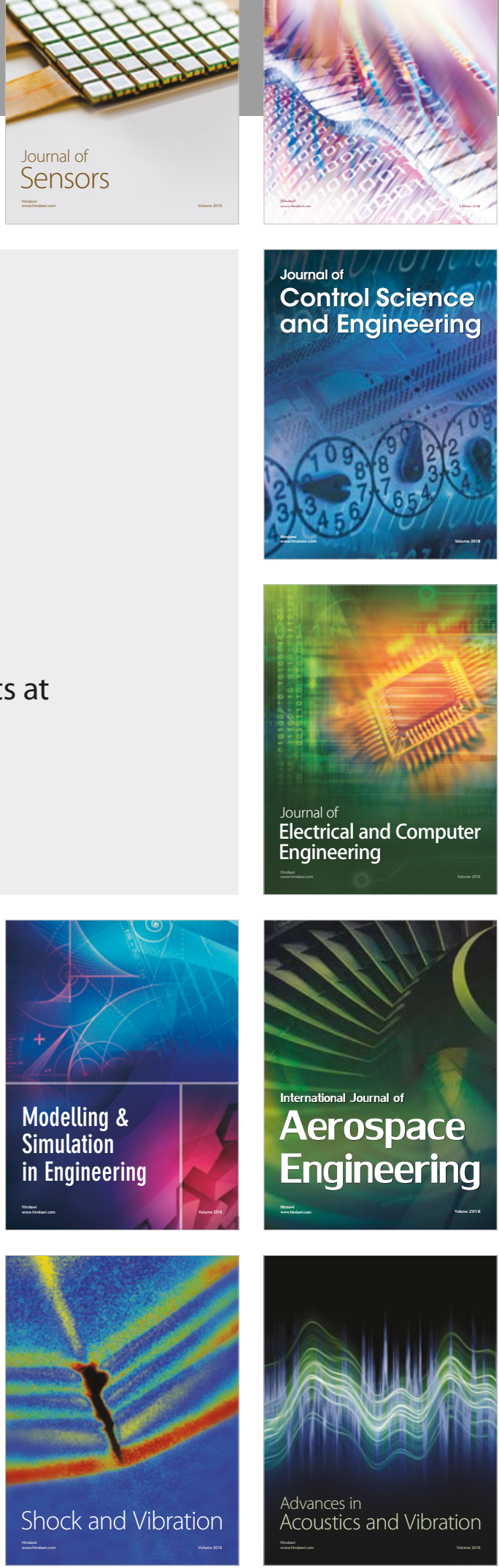